\title{
AGN and Galaxy Clustering at $z=0.3-3.0$ Using the Japanese Virtual Observatory
}

\author{
Yuji Shirasaki ${ }^{1}$, Masahiro Tanaka ${ }^{2}$, Masatoshi Ohishi ${ }^{1}$, \\ Yoshihiko Mizumoto $^{1}$, Tadafumi Takata ${ }^{1}$, and Naoki Yasuda ${ }^{3}$ \\ ${ }^{1}$ National Astronoimcal Observatory of Japan, \\ 2-21-1 Osawa, Mitaka, Tokyo, Japan \\ ${ }^{2}$ University of Tsukuba, \\ 1-1-1 Tennodai, Tsukuba, Ibaraki, Japan \\ ${ }^{3}$ Institute for the Physics and Mathematics of the Universe Library, The University of Tokyo, \\ 5-1-5 Kashiwa-no-ha, Kashiwa, Chiba, Japan
}

Keywords. large-scale structure of universe, quasars: general, galaxies: evolution, galaxies: formation, astronomical data bases: miscellaneous

We present the results of a cross-correlation analysis of the projected positions of AGNs and galaxies at redshifts from 0.3 to 3.0. It is widely accepted that the origin of AGN activity is accretion of matter onto a massive black hole at the center of a galaxy (e.g., Lynden-Bell 1969). To explain the activity of AGNs, a large fraction of matter in the galaxy must be delivered to the inner region on a short timescale (Hopkins et al. 2008). One possible mechanism for causing rapid gas inflows into the central region is a major galaxy merger between gas-rich galaxies (e.g., Kauffmann \& Haehnelt 2000). If this is the case, AGNs are expected to be found in an environment with higher galaxy density than that of typical galaxies. We investigated environments of $\sim 750$ AGNs, which is about a ten times larger sample than used in previous studies, and we find a significant excess of galaxies around the AGNs in the redshift range of 0.3 to 1.8. We used the Japanese Virtual Observatory (JVO) to obtain the Subaru Suprime-Cam images and UKIDSS data around known AGNs. The datasets accessed through the JVO are: Catalog of Quasars and Active Galactic Nuclei by Veron-Cetty et al. (2006), SDSS DR-5 Quasar Catalog by Schneider et al. (2007), Subaru Suprime-Cam Reduced Image Archive of JVO, and UKIDSS DR2 catalog by Warren et al. (2007). We divided all the AGN samples into four redshift groups, $0.3 \lesssim z \lesssim 0.8,0.8 \lesssim z \lesssim 1.5,1.5 \lesssim z \lesssim 1.8$, and $1.8 \lesssim z \lesssim 3.0$. For each redshift group, the dataset was further divided into a fainter group $\left(M_{V} \geqslant-25 \mathrm{mag}\right)$ and a brighter group $\left(M_{V}<-25 \mathrm{mag}\right)$. We found that the correlation length of the high-redshift bright sample $(1.5 \lesssim z \lesssim 1.8)$ was larger than that of the low-redshift faint sample $(0.3 \lesssim z \lesssim 0.8)$. We also found that the correlation length was larger for the faint group at redshift range $0.8 \lesssim z \lesssim 1.5$. These results can be explained by downsizing of mass assembly. More details can be found in the paper by Shirasaki et al. (2009). Our result implies that the Japanese Virtual Observatory can be a powerful tool to investigate the co-evolution of central black holes and galaxies at the intermediate redshift universe.

\section{References}

Hopkins, P. F., Hernquist, L., Cox, T. J., \& Keres, D., 2008, ApJS, 176, 356

Kauffmann, G. \& Haehnelt, M. G., 2000, MNRAS, 311, 576

Lynden-Bell, D. 1969, Nature, 223, 690

Schneider, D. P., et al., 2007 AJ, 134, 102

Shirasaki, Y. et al. 2009, submitted to PASJ [arXiv:0907.5380]

Veron-Cetty, M.-P. \& Veron, P. A\&A, 455, 773

Warren, S. J., et al. 2007 [arXiv:astro-ph/0703037] 